\title{
The study of the safety importance analysis method on A / B classification for periodic test surveillance
}

\author{
Xiao-Yu Liang, Zi-Jun Yang and Liang Mei \\ Suzhou Nuclear Power Research Institute, \\ Shenzhen, Guangdong, China \\ tE-mail: liangxiaoyu@cgnpc.com.cn \\ www.snpi.ac.cn
}

\begin{abstract}
Currently, the Periodic Test Surveillance has too much test items with different importance, and there is no different management requirement for the test items with different safety affect. The method described in this article can divided these test items in importance(mark A) and non-importance (mark B).This method is an integrated evaluation method, that is deterministic impact analysis as based, supplemented by probabilistic safety assessment method. This ensures accuracy and reasonableness of evaluation results.
\end{abstract}

Keywords: Periodic Test Surveillance; Safety importance analysis; A / B Classification;

\section{Introduction}

The content of the Periodic Test Surveillance is determined by the method which is an expansion of the safety function as the core. This method makes the Periodic Test Surveillance cover all system, equipment and parts of all security function, other functions as much as possible. So, it can ensure all the safety function completely covered by it. To some extent, this method can ensure the integrity of the content, but also led to a safety and economy issues because of the no distinction management.

For this Periodic Test Surveillance, the safety affected of the test items vary greatly, and there is no different management requirement for the different test items with different safety affect, so it is necessary for the test terms in the Periodic Test Surveillance to do some safety classification (A/B Group).On this basis, it can realize the aim of safety management optimization.

\section{Process of the Criteria Classification for Nuclear Power Plant Periodic tests Surveillance Program}

The work of the test terms A/B classification includes two steps (see Figure 1).The first step is to set up the classification standard [1], and the second step is 
to dothe completely safety analysis for every test item with the deterministic analysis and the probabilistic analysis.

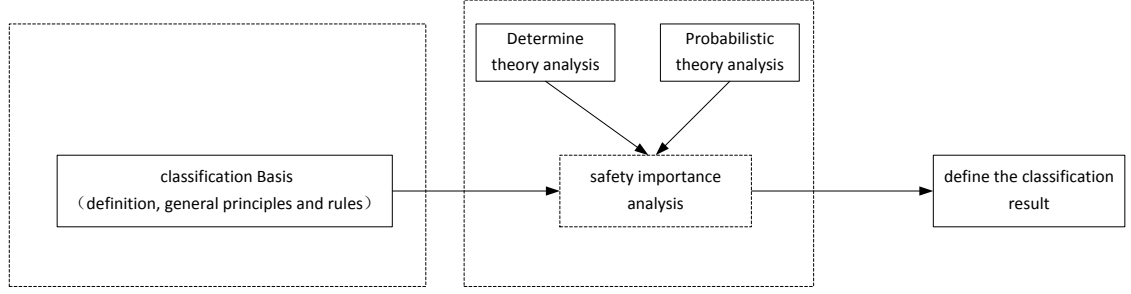

Fig. 1. Process of the classification for every test item.

\section{Introduction of the Importance Analysis method}

The safety importance analysis is the key element to the criterion classification accuracy. This article is studying about the method used to do the safety importance analysis, including Deterministic theory analysis and Probabilistic theory analysis. Also make some examples for these two methods. These two methods can avoid the risks of failure analysis, so it can ensure the accuracy of test item classification.

\subsection{Deterministic analysis and example}

In the design, The Technical Specification[2] and The Periodic Test Surveillance[3] are part of regulatory documents of nuclear power plants. The Technical Specification includes two aspects: 1.Safety limits during normal operation; 2.System and Equipment used to limit the consequences of the accident.

The content of The Periodic Test Surveillance is test items about the two aspects in The Technical Specification. Through The Periodic Test Surveillance, we can make sure the availability of equipment and system in The Technical Specification.

Based on the writing principle of The Technical Specification, we divided the function and equipment into five categories from the perspective of security features, namely:

Category 1: Initial conditions of the design basis accident(DBA) (thermal, neutron parameters).

Category 2:System and equipment acquired in the automatic operation phase for the design basis accidents (including support system).

Category 3: Systems and equipment acquired to achieve and maintain safe shutdown condition for the design basis accidents (operator control phase in the accident research). 
Category 4: Hypothesis, Systems and equipment acquired in the supplementary analysis accidents.

Category5: system and equipment related to the three barrier integrity or radioactive shielding.

According to the consequences when the above five categories are not complied with, we establish the principle of classification as follows(Figure 2):

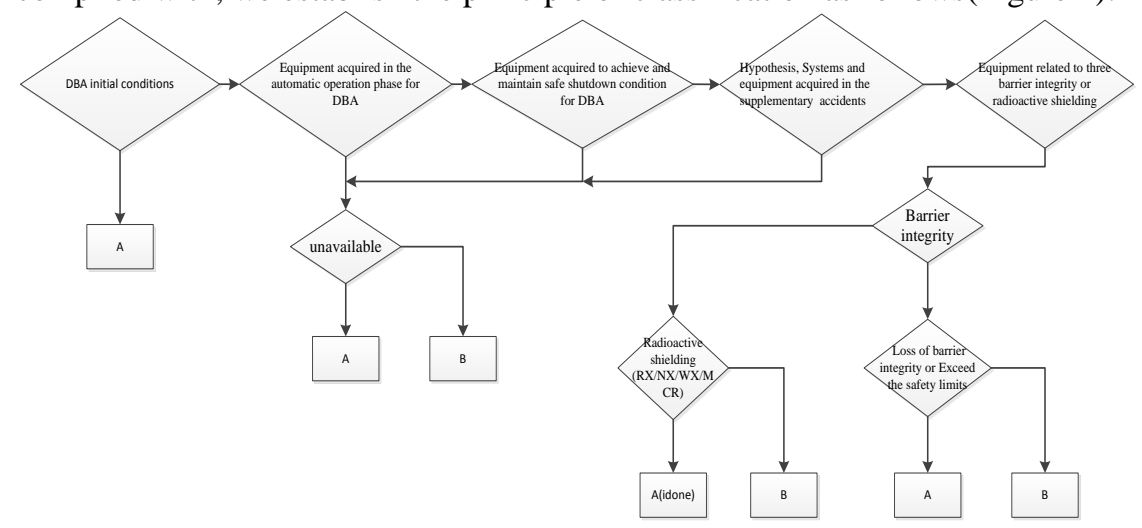

Fig. 2. Principle of classification.

The method of using the Deterministic theory to do the safety importance analysis is mainly about the analysis of what the security functions are, what kind of performance requirements to be achieved for carrying out these security features, what the impact is on the safety function exactly if the test term is not satisfied[4].

How to make sure the accuracy of this method in the safety importance analysis? It mainly depends on the analyst knowledge on the security features or devices. This method needs analysis and design staff for these functions or equipment with sufficient knowledge and understanding, also needs to refer to the corresponding design file or safe files.

By summarizing these five categories needed to strengthen supervision, we can draw a conclusion about what corresponding file can be refer to during do the safety importance analysis. For example, for safety function analysis, you can refer to the relevant documents about security analysis, such as Final Safety Analysis Report (FSAR), System Security Guidelines (SSC); for accident influence analysis, you can refer to the accident procedures; for the equipment or system availability analysis, you can refer to system-related design documents system Design Manual (SDM), Equipment Operation and Maintenance Manual. In addition to these documents, we also need to refer to the up-level document of the Periodic Test Surveillance, such as the Periodic Test Completeness Note (PTCN) and the Periodic Test Rule(PTR). 
Example: "system integrated test/simulation PTR in low level 3 situation and check the EAS recirculation sequence: the correct order

Deterministic theory analysis: The EAS security function is to reduce the temperature and pressure in the containment and iodine concentration, and to remove heat away from the containment when are under the accident conditions (such as LOCA or containment vessel steam pipes burst).

When PTR001BA appeared low level 3, EAS needs to turn to turn to the recycle mode, in order to ensure the EAS pump suction flow rate.

If the test item does not meet the requirements, it will affect the realization of the function of containment spray.

According to the Category 2, this test item is about System acquired in the automatic operation phase for the design basis accidents and the test item does not meet the requirement will cause unavailable. So we divided it into Group A.

\subsection{Probabilistic analysis and example}

Nuclear power plant real-time risk assessment and management (RM) is based on PSA models and methods, and make the unit configuration as input information. It can easily and quickly evaluate the risk level of the power plant in the production activities (Operation, test, maintenance, etc.), and provide riskinformation for decision-making during operation, testetc[5].

For some test items, because of its relatively local inspection, when such a test item is not respected, the overall safety impact assessment of the equipment is not clear. Under such conditions, we can use RM to assess the risk and make the results as an auxiliary for the safety importance analysis.

Example: "ASG turbine steam supply line availability / ASG131VV132 VV-133VV availability: the rotational speed is normal"

Deterministic theory analysis: ASG131VV-132VV-133VV are 3 check valves on the steam generator line, upstream of the main steam isolation valves. They are the interface to the turbine extraction steam. When there are one or two valves unavailable, it does not affect the Steam Pump ASG003PO availability, but will no longer meet the single failure criterion. If the test item does not meet the criterion, it will not affect the availability of Steam Pump ASG003PO.

Probability Theory Analysis: Evaluated three cases by RM:1.only one check valve is not available (such as ASG131VV),2.any two check valves are not available (such as ASG131VV and ASG132VV),3.All the three check valve are unavailable. Through the evaluation results can be seen: the case of a check valve is not available or any two check valves are not available is lead to core damage frequency is $1.29 \mathrm{E}-05$ / reactor years, the risk level is low, in this configuration permits duration of more than 30 days; when all three check valve are not available, it can result the core damage frequency to be 7.27E-05 / 
reactor years, the risk level is medium, in this configuration allows a duration of two days 16 hours.

Thus, any one or two check valve is unavailable, there is not affect to the Steam Pump ASG003PO availability.

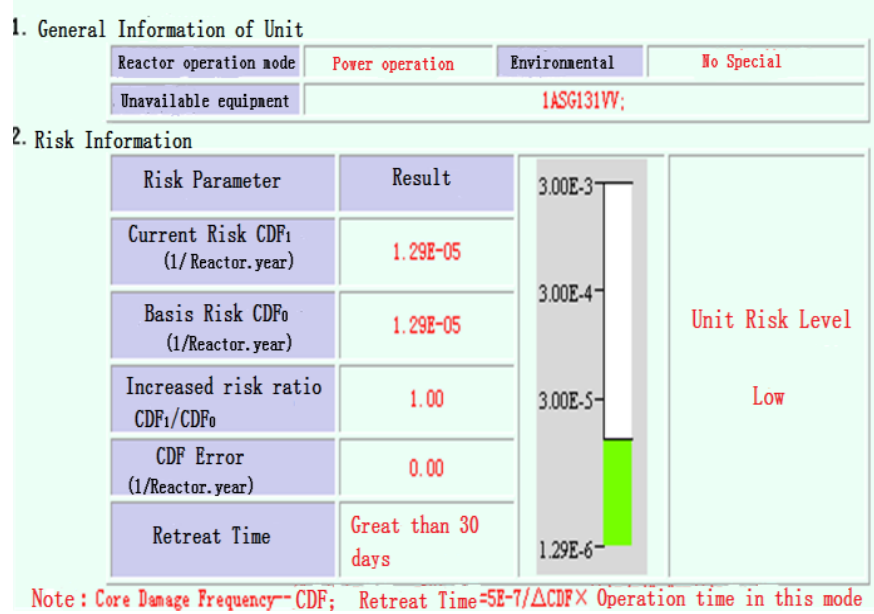

Fig. 3. ASG131VV unavailable.

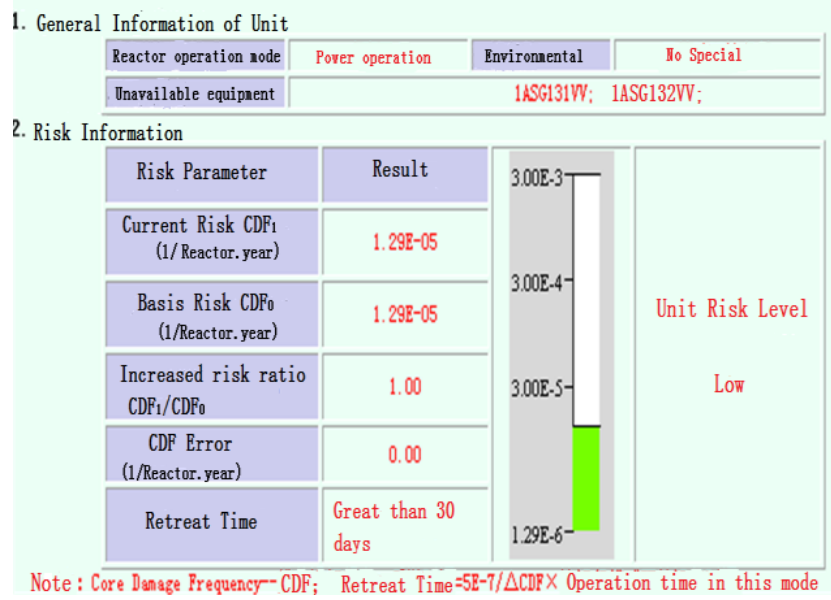

Fig. 4. ASG131VV-132VV unavailable. 


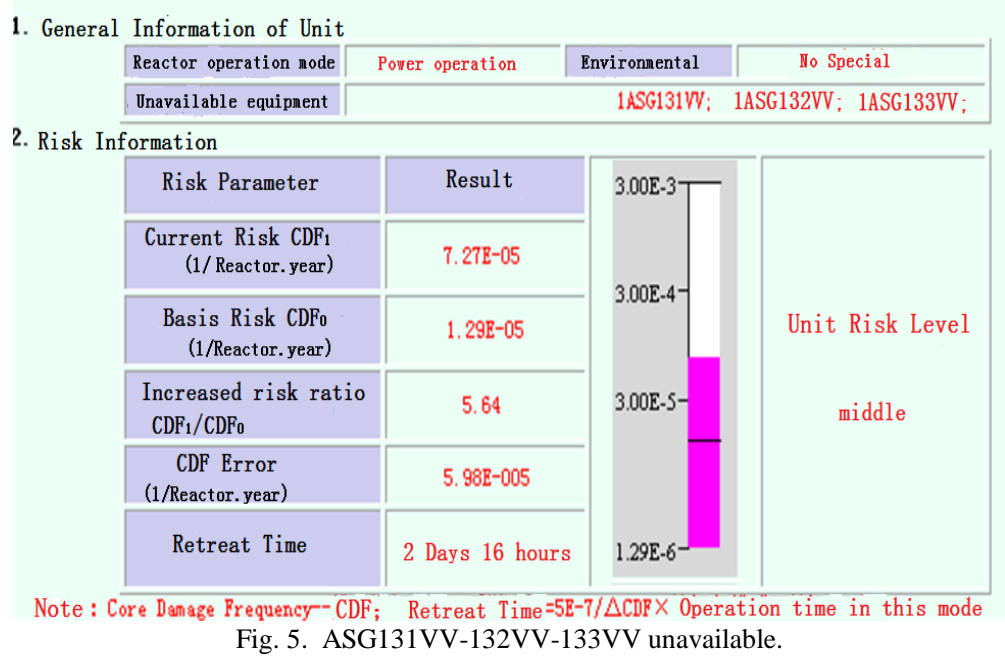

\section{Conclusion}

The deterministic analysis is a qualitative analysis and requires the analysts fully understanding of the systems and equipment, and it also requires the classification principles must be comprehensive and accurate, in order to ensure the accuracy of the classification results.

The probability analysis can carry out a quantitative analysis for the test item and the result can be intuitive. But it depends on the details of the PSA model, as our PSA model is not enough detailed to cover every test item, so it is unrealistic to use the probability analysis to do the important analysis for every item in The Periodic Test Surveillance

For safety importance analysis, we can use the comprehensive evaluation method, which is based on the deterministic analysis and the probability analysis supplemented.

\section{References}

1. Yang Zijun, Guidelines of DNMC $<$ Safety-related systems and equipment Periodic test monitoring requirements>A/B classification Project,2015.(In Chinese)

2. Yao Zhigang, Technical Specifications(Ling Ao Nuclear Power Plant Unit 3/4), 2013.(In Chinese)

3. Liu Wenyuan, 《Periodic test surveillance requirement of the safety-related systems and equipment》(Ling Ao Nuclear Power Plant Unit 3/4), 2015.(In Chinese) 
4. Qiu Bin, Criteria classification management analysis for periodic test surveillance program in Ling Ao Nuclear Plant,2009.(In Chinese)

5. IAEA, Application of probabilistic safety assessment(PSA) for nuclear power plants,IAEA-TECDOC-1200.Feb.2001 